\title{
Comparison of Musculocutaneous and Fasciocutaneous Free Flaps for the Reconstruction of the Extensive Composite Scalp and Cranium Defects
}

\author{
Hakan Uzun, MD, ${ }^{*}$ Ozan Bitik, MD, ${ }^{\dagger}$ Umut Sinan Ersoy, $M D,^{\ddagger}$ \\ Burçak Bilginer, $M D{ }^{\S}$ and Ali Emre Aksu, MD*'
}

\begin{abstract}
Composite scalp and cranium defects, which require microsurgical reconstruction, result from tumor resection, radiation, trauma, severe burn injuries, and rarely vasculitic disorders. In the current study, the authors aim to compare the outcomes of the fasciocutaneous flaps and musculocutaneous free flaps used for the reconstruction of extensive composite scalp and cranium defects. From 2010 to 2017,21 patients who underwent composite scalp and cranium defect reconstruction with a free flap were retrospectively identified. Eighteen patients had squamous cell carcinoma, 2 patients had meningioma, and 1 patient had Ewing sarcoma. Thirteen musculocutaneous free flaps including latissimus dorsi and vertical rectus abdominis flaps and 9 free fasciocutaneous flaps including radial forearm and anterolateral thigh flaps were used. Only 1 flap loss was encountered. No neurologic impairment in postoperative period was reported. The mean length of stay in the hospital, the duration of surgery, and total volume of blood transfusion for the fasciocutaneous flap group were significantly shorter than those for musculocutaneous flap group. No flap atrophy was reported in fasciocutaneous flap group. Reconstruction of the composite scalp and cranium defects with fasciocutaneous free flaps allows shorter hospitalization, less blood transfusion and less flap atrophy than those of musculocutaneous flaps. To this respect, their usage should be prioritized in such challenging patients.
\end{abstract}

Key Words: Craniectomy, free flap, scalp reconstruction

(J Craniofac Surg 2018;29: 1947-1951)

From the *Department of Plastic, Reconstructive and Aesthetic Surgery, Hacettepe University Faculty of Medicine, Sihhiye; †Plastic, Reconstructive and Aesthetic Surgery, Next Level, Ankara; $\ddagger$ Department of Plastic, Reconstructive and Aesthetic Surgery, Acibadem University Faculty of Medicine, Küçükçekmece, Istanbul; and §Department of Neurosurgery, Hacettepe University Faculty of Medicine, Ankara, Turkey.

Received April 13, 2018.

Accepted for publication August 17, 2018.

Address correspondence and reprint requests to Hakan Uzun, MD, Department of Plastic, Reconstructive and Aesthetic Surgery, Hacettepe University Faculty of Medicine, 06100 Sihhiye, Ankara, Turkey; E-mail: shakanuzuns@gmail.com

This study was presented at the 4th Congress of Asian Pacific Federation of Societies for Microsurgery in Antalya, Turkey, May 9 to 13, 2018.

The authors report no conflicts of interest.

Copyright 102018 by Mutaz B. Habal, MD

ISSN: $1049-2275$

DOI: $10.1097 /$ SCS.0000000000005052 omposite scalp and cranium defects, which require microsurgical reconstruction, result from tumor resection, radiation, trauma, severe burn injuries, and rarely vasculitic disorders. ${ }^{1-3}$ These soft tissue and bone defects are distinct with regard to anatomy, complication rates, and reconstructive options. Scalp and cranium protect the underlying dura and brain. Once this protection is lost, the patients face the risks of meningitis, brain abscess, cerebrospinal fluid (CSF) leak, and seizures. Prior or planned radiotherapy makes the scenario even worse.

Local or regional flaps generally fall short given the fact that the defects created are generally beyond these options. Staged approach using tissue expansion is not feasible due to malignant nature of the process. Free flaps strengthen the plastic surgeons' hand and bring diversity to the reconstruction alternatives. Many techniques have been defined in the literature so far and most of them comprise the same surgical principals. ${ }^{4-8}$ The advantages of free flap reconstruction depend on various factors including but not limited to etiology and size of the defect, donor site morbidity, and involvement of surrounding structure. ${ }^{9}$ Controversies exist to decide a cranioplasty should be added.

In the current study, the authors aim to share their experiences and to compare the outcomes of the fasciocutaneous flaps and musculocutaneous flaps used for the reconstruction of extensive composite scalp and cranium defects.

\section{MATERIALS AND METHODS}

The current study was conducted according to the Declaration of Helsinki. Informed consent was obtained from each patient. From 2010 to 2017, 21 patients who underwent composite scalp and cranium defect reconstruction with a free flap were retrospectively identified. The medical records of patients were reviewed for demographics, tumor pathology, defect location, flap choice, length of stay in hospital, and complications. Patients with a minimum postoperative follow-up of 12 months were included for the study. Patients were excluded from the study if they had a reconstruction other than a free flap, if they had only scalp defect reconstructed with a free flap, if they had only split thickness cranium defect or if they had combined defects of the periorbita and/or midface regions. The patients meeting these criteria were grouped into 2 categories according to the composition of the flap: fasciocutaneous or musculocutaneous.

\section{Surgical Procedure}

Initially, the resection of tumor or the debridement of nonviable tissue was undertaken. The cutaneous malignancy was resected with 1 to $2 \mathrm{~cm}$ surgical margins. The degree and extent of cranium involvement was determined by both clinically and radiologically. Trying to have $1 \mathrm{~cm}$ oncologically safe bone margin, a circular craniectomy was performed. Dura was removed by the neurosurgery team. Duraplasty with fascia lata graft was added if brain parenchyma was exposed. Brain parenchyma resection was also 
decided by neurosurgery team. The neck dissection, when necessary, was performed. The harvest of the flap was initiated once we were sure of that the margins were tumor-free or all the necrotic scalp and bone were removed in patient with osteoradionecrosis. Recipient vessels for anastomosis were then prepared. The main vessels of choice were superficial temporal vessels. In patient with venous insufficiency, we explored the neck for any suitable vein.

\section{Statistical Analysis}

Statistical analysis of the data was performed by using SPSS 17 (SPSS Inc, Chicago, IL). The length of stay in the hospital, duration of the surgery, amount of blood transfusion, flap atrophy leading to wound dehiscence, and complications were compared between fasciocutaneous and musculocutaneous reconstruction groups by using a $t$ test. $P \leq 0.05$ was considered significant.

\section{RESULTS}

\section{Demographic Findings}

Twelve of the patients were males and 9 were females. Average patient age was 54.9 years (range from 15 to 82 years). No significant difference existed between 2 groups in terms of age (Table 1). The mean follow-up time was 37 months (13-64 months). Three of the patients had previous unsuccessful closure attempts to scalp.

\section{Tumor Characteristics}

Eighteen patients had squamous cell carcinoma (SCC), 2 patients had meningioma, and 1 patient had Ewing sarcoma. One of the patients with meningioma was operated because of exposure of alloplastic implant, which was implanted 2 years ago during tumor removal (Fig. 1). One of the patients with SCC was operated because of osteoradionecrosis of the cranium, which was caused by previous high-dose radiotherapy (Fig. 2). Remaining 19 patients had tumor invasion of both skin and cranium. Twelve patients with SCC had parenchymal involvement. Seven patients had prior radiotherapy and 8 patients took postoperative radiotherapy (Table 2 ).

\section{Flap and Reconstruction Characteristics}

Twenty-two free flaps were performed for scalp and cranium reconstruction (Tables 1-2). Four different types of free flaps including radial forearm flap (RFF) (Fig. 3), anterolateral thigh (ALT) flap (Fig. 4), vertical rectus abdominis musculocutaneous

\begin{tabular}{lcc}
\hline TABLE 1. Comparison of Both Groups According to Statistical Parameters \\
\hline & $\begin{array}{c}\text { Fasciocutaneous } \\
\text { Flap }\end{array}$ & $\begin{array}{c}\text { Musculocutaneous } \\
\text { Flap }\end{array}$ \\
\hline Age & $56.1(25-82$ y) & $54.6(15-78 \mathrm{y})$ \\
Sex & 6 Males, 3 females & 8 Males, 4 females \\
Cranium reconstruction with & - & 1 (methyl methacrylate) \\
$\quad$ alloplastic implantation & $155 \pm 40$ & $236.4 \pm 62$ \\
Blood (erythrocyte suspension) & & $16.7 \pm 4.5$ \\
$\quad$ transfusion, mL & $12.4 \pm 3.1$ & $11.3 \pm 2.6$ \\
Hospitalization, d & $8.6 \pm 1.2$ & $4(36.4 \%)^{*}$ \\
Duration of surgery, $h$ & - & 1 \\
Flap atrophy & 6 & 8 \\
Skin grafting of donor site & - & \\
Skin grafting over rough & & \\
$\quad$ surface of flap & & \\
\hline
\end{tabular}

*No wound dehiscence due to flap atrophy was encountered.

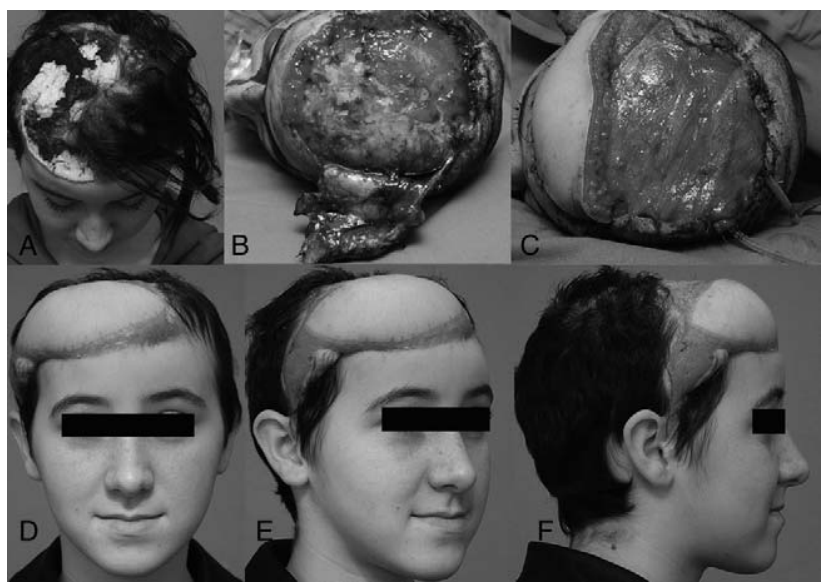

FIGURE 1. (A) Preoperative appearance of 15-year-old female patient with an exposed alloplastic material, which was implanted 2 years ago during meningioma removal. Dura was exposed. (B) Intraoperative appearance after removal of exposed implant. (C) Free latissimus dorsi flap covered the composite defect. (D-F) Postoperative 6th-month appearances.

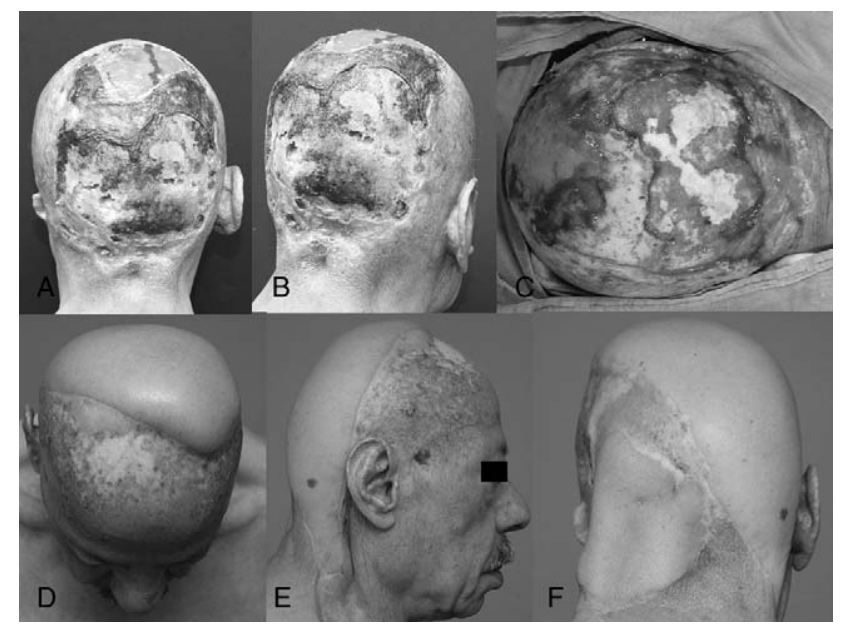

FIGURE 2. (A, B) Preoperative appearance of 71-year-old male patient with osteoradionecrosis of the scalp and cranium. The brain parenchyma was visible through the defect. (C) Radionecrotic bone was aggressively removed. (D-F) Postoperative 2 nd-year appearances after the defect was reconstructed with a free latissimus dorsi flap. Transverse cervical vessels were used for microanastomes due to previous radiotherapy and sacrifice of both superficial temporal vessels. The nape of the neck was reconstructed with a pedicled trapezius musculocutaneous flap.

(VRAM) flap, and latissimus dorsi musculocutaneous (LD) flap (Fig. 5) were accomplished. Thirteen musculocutaneous flaps and 9 fasciocutaneous flaps were used. In 4 patients with LD flap reconstruction, skin island was designed in such a way that it covered thoracodorsal vessels (Figs. 2 and 5). Transverse cervical vessels were chosen for the patient with osteoradionecrosis of the scalp because of prior radiotherapy and sacrifice of both superficial temporal arteries (Fig. 2). Only 1 flap loss was encountered. All of the rough surfaces of the musculocutaneous flaps were skin grafted 3 weeks later. No cranioplasty was intended except for 1 patient with recurrent meningioma within the posterior cranial fossa (Fig. 6).

\section{Hospitalization}

The mean length of stay in the hospital following surgery for the fasciocutaneous flap group was significantly shorter than that of the 


\begin{tabular}{|c|c|c|c|c|c|}
\hline Primary Tumor & Flap & Defect Size (Scalp), cm & Defect Size (Cranium), cm & Defect Location & Radiotherapy \\
\hline SCC & LD & $20 \times 10$ & $6 \times 6$ & Parietal & Postoperative \\
\hline SCC & LD & $20 \times 10$ & $8 \times 4$ & Frontoparietal & Postoperative \\
\hline SCC & $\mathrm{LD}$ & $18 \times 12$ & $8 \times 6$ & Frontoparietal & Preoperative \\
\hline SCC & $\mathrm{LD}$ & $21 \times 11$ & $10 \times 8$ & Frontoparietal & Preoperative \\
\hline SCC & VRAM & $18 \times 10$ & $8 \times 8$ & Frontoparietal & - \\
\hline SCC & VRAM $^{*}$ & $19 \times 10$ & $10 \times 6$ & Frontoparietal & - \\
\hline SCC & ALT & $16 \times 12$ & $10 \times 7$ & Temporoparietal & Postoperative \\
\hline SCC & ALT & $15 \times 10$ & $11 \times 5$ & Parietal & Postoperative \\
\hline SCC & ALT & $14 \times 10$ & $11 \times 6$ & Temporoparietal & - \\
\hline SCC & ALT & $18 \times 15$ & $8 \times 7$ & Temporoparietal & Preoperative \\
\hline Ewing sarcoma & LD & $18 \times 12$ & $10 \times 10$ & Parietooccipital & Postoperative \\
\hline SCC & $\mathrm{RF}$ & $10 \times 10$ & $6 \times 6$ & Temporoparietal & Postoperative \\
\hline $\mathrm{SCC}$ & $\mathrm{RF}$ & $14 \times 8$ & $9 \times 6$ & Parietal & Preoperative \\
\hline $\mathrm{SCC}$ & LD & $15 \times 8$ & $8 \times 6$ & Parietooccipital & - \\
\hline SCC & $\mathrm{RF}$ & $12 \times 7$ & $7 \times 5$ & Parietal & - \\
\hline SCC & LD & $18 \times 15$ & $12 \times 9$ & Parietal & Postoperative \\
\hline Meningioma & LD & $15 \times 15$ & $12 \times 12$ & Parietooccipital & - \\
\hline SCC & $\mathrm{RF}$ & $12 \times 8$ & $7 \times 5$ & Temporoparietal & Preoperative \\
\hline $\mathrm{SCC}$ & $\mathrm{RF}$ & $16 \times 8$ & $8 \times 5$ & Temporoparietal & Preoperative \\
\hline SCC & LD & $16 \times 10$ & $12 \times 7$ & Frontoparietal & Postoperative \\
\hline Meningioma & $\mathrm{LD}$ & $18 \times 12$ & $12 \times 8$ & Frontoparietal & Preoperative \\
\hline
\end{tabular}

ALT, anterolateral thigh; LD, latissimus dorsi musculocutaneous; RF, radial forearm; SCC, squamous cell carcinoma; VRAM, vertical rectus abdominis musculocutaneous. ${ }^{*}$ VRAM flap was lost and LD free flap was performed as salvage procedure.

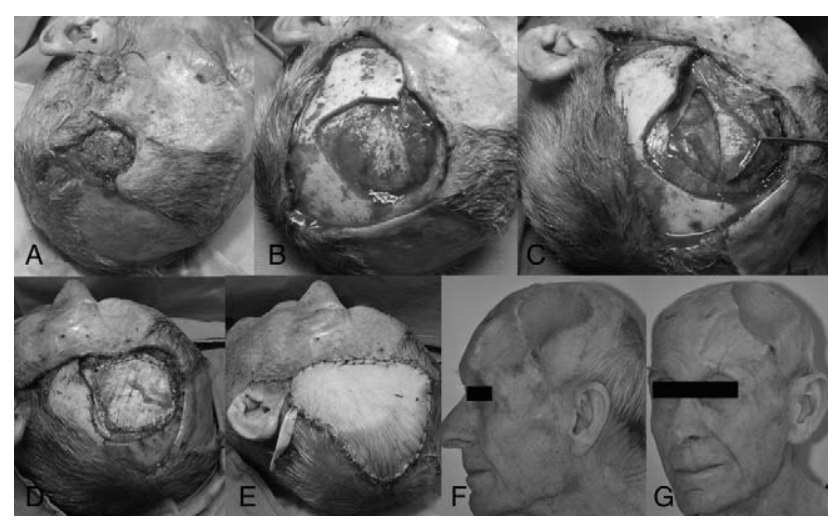

FIGURE 3. (A) Preoperative appearance of 82-year-old male patient with recurrent squamous cell carcinoma invading dura. (B) Intraoperative appearance after resection of scalp and cranium. (C) Intraoperative appearance during dura removal showing full-thickness dural invasion. (D) Intraoperative appearance after duraplasty with fascia lata graft was completed. (E) The defect was reconstructed with free radial forearm flap. $(F, G)$ Postoperative 1-year appearances.

musculocutaneous flap group $(12.4 \pm 3.1$ days vs $16.7 \pm 4.5$ days, respectively; $P<0.05$ ) (Table 2 ). Cumulative hospitalization involving the primary reconstructive procedure and secondary procedures like skin grafting and flap reshaping were also significantly higher in musculocutaneous flap group (14.9 \pm 2.7 days vs $21.3 \pm 3.6$ days, respectively; $P<0.05$ ).

\section{Complications}

Neither dural fistula nor CSF leakage was seen. We did not encounter any neurologic impairment in postoperative period. Two patients had venous outflow problem in the immediate

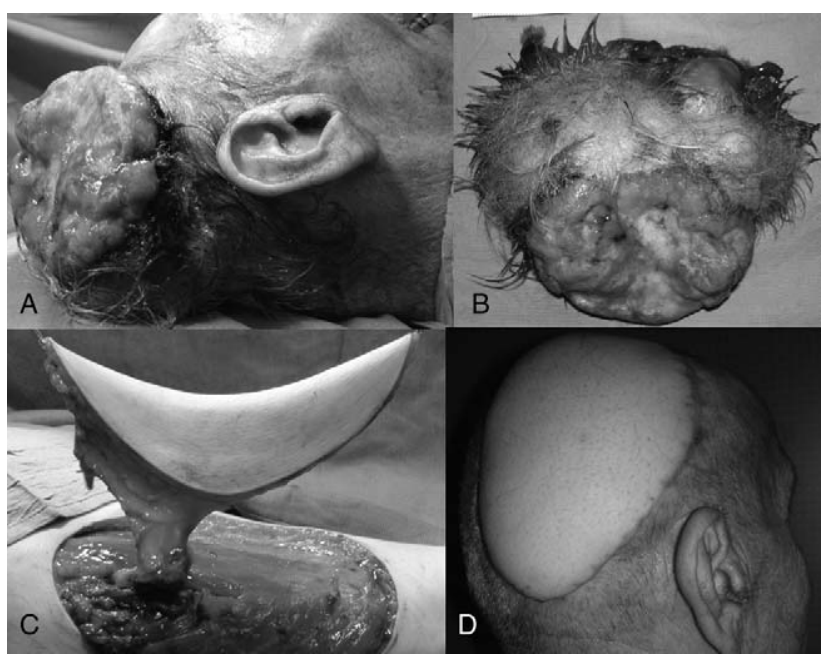

FIGURE 4. (A) Preoperative appearance of 78-year-old male patient with squamous cell carcinoma invading cranium in the parietal region. (B) $A$ $15 \times 10 \mathrm{~cm}$-tumor was resected. (C) The defect was reconstructed with free anterolateral thigh flap. (D) Postoperative 18th-month appearance.

postoperative period and were taken back to the operating theater for venous reanastomosis with a vein graft to external jugular vein. We observed 2 skin graft lyses and 2 seroma formations at the donor site in both groups. The amount of blood transfusion in the form of erythrocyte suspension was significantly higher in musculocutaneous flap group $(P<0.05)$ (Table 2$)$. During 7-year follow-up, 3 patients died because of distant metastasis and 1 patient died because of a myocardial infarction (Table 3).

According to the experiences from this series, we devised a simple algorithm prioritizing free fasciocutaneous flaps for the reconstruction of extensive scalp and cranium defects (Fig. 7). 


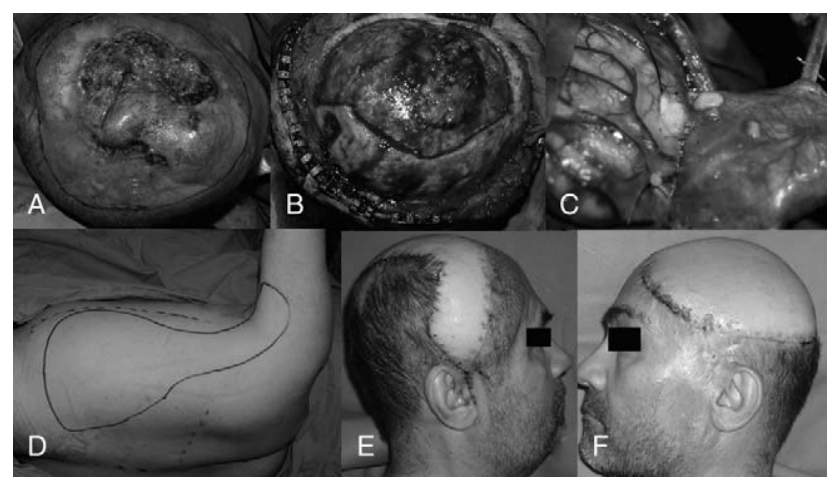

FIGURE 5. (A) Preoperative appearance of 58-year-old male patient with recurrent squamous cell carcinoma invading dura. (B) Intraoperative appearance after resection of scalp and cranium. (C) Intraoperative appearance during dura removal showing full-thickness dural invasion. (D) Free latissimus dorsi flap covered the composite defect. The flap was designed in such a way that the skin island covered thoracodorsal vessels. (E, F) Postoperative 3rd-week appearances.

TABLE 3. Complications

\begin{tabular}{lcc}
\hline & $\begin{array}{c}\text { Fasciocutaneous } \\
\text { Flap }\end{array}$ & $\begin{array}{c}\text { Musculocutaneous } \\
\text { Flap }\end{array}$ \\
\hline Microanastomosis revision & - & 2 \\
Flap loss & - & 1 \\
Infection & - & - \\
Wound healing problem in scalp & 2 (skin graft lysis) & 2 (seroma) \\
Wound healing problem in donor site & - & - \\
Dural fistula & - & - \\
Neurologic impairment & 2 & 2 \\
Mortality & &
\end{tabular}

\section{DISCUSSION}

In the current study, patients with fasciocutaneous flaps had significantly shorter duration of hospitalization than the patients with musculocutaneous flaps. When compared to each other, RFF and ALT flap showed no significant difference in terms of duration of hospitalization. In their large series, Sweeny et $\mathrm{al}^{9}$ showed that

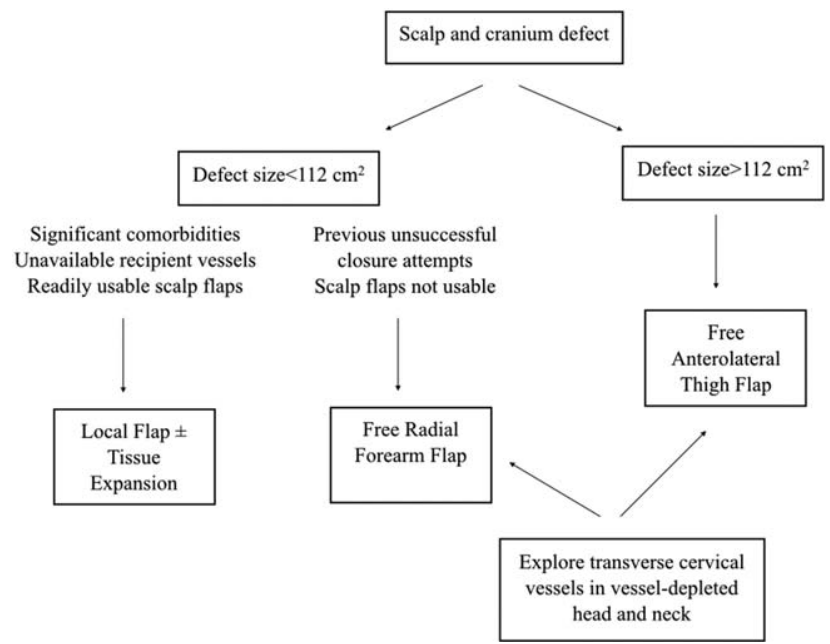

FIGURE 7. Proposed reconstructive algorithm of extensive composite scalp and cranium defects.

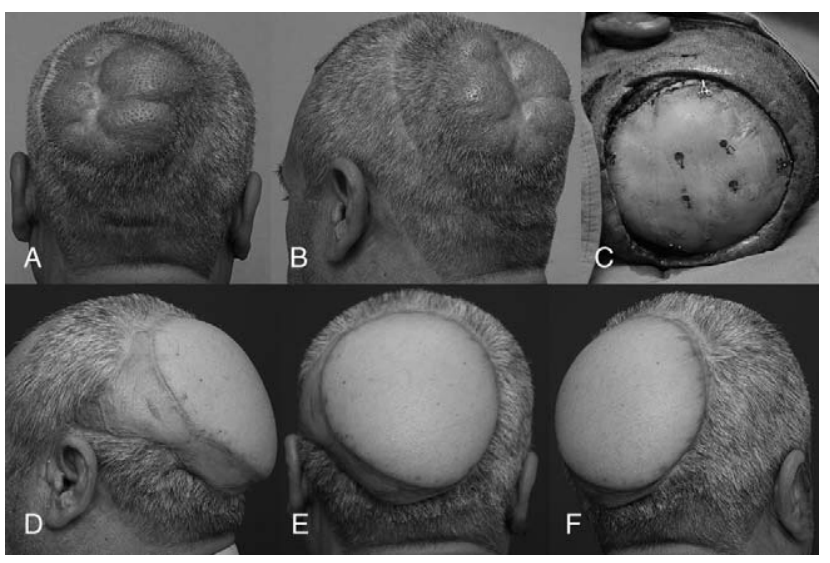

FIGURE 6. (A, B) Preoperative appearance of 54-year-old male patient with recurrent meningioma. (C) Following tumor removal, craniectomy defect was reconstructed with an alloplastic material by neurosurgery team. (D-F)

Postoperative 1-year appearances after the defect over the implant was reconstructed with a free latissimus dorsi flap. Rough surface of the flap was skin grafted 3 weeks later.

radial forearm free flap was associated with few complications and shorter duration of hospitalization when compared to LD and VRAM flaps. They used radial forearm free flap more often in the frontal region whereas we used it in the parietal region predominantly. In contrary to our results, they concluded that choice of microsurgical free flap did not affect the length of hospitalization.

Regarding the flap choice, a clear advantage of fasciocutaneous flaps was the shorter duration of surgery. The major reasons to use a fasciocutaneous flap in our series were relative ease of flap harvest, allowing 2-surgical team approach and no need for repositioning the patient unlike an LD flap would need. In a study comparing the outcomes of younger and older patients requiring scalp reconstruction with free LD and ALT flaps, Simunovic et $\mathrm{al}^{10}$ found no difference between groups. They implied that duration of surgery was reduced when ALT flap was used. Although duration of surgery does not seem to be related with perioperative complications in our series, it clearly decreases the frustration of the microsurgeon.

Another advantage of fasciocutaneous flaps was that they are prone to less atrophy. Flap atrophy generally results from denervation of muscle. In addition, postoperative radiotherapy itself may cause flap atrophy or even may worsen it. In our series, we encountered flap atrophy only in the musculocutaneous flap group although it did not cause any late complications. Sosin et al ${ }^{1}$ reported 3 patients with free flap atrophy following scalp and calvarium reconstruction. Two of them were fasciocutaneous ALT and ulnar forearm flaps and the remaining one was LD flap. However, they mainly observed flap atrophy in patients with previous unsuccessful reconstruction attempts with LD flap. Others reported that ALT flap underwent less atrophy than the LD flap and for that reason they shifted their main flap choice for scalp reconstruction over the years. ${ }^{11}$

Radial forearm flap was the most rewarding flap in terms of blood loss, hospitalization, and duration of the surgery. Its main constraint comes from the enormousness of the defect size. In our series, if the dimension of the scalp defect was more than $112 \mathrm{~cm}^{2}$, another free flap option was taken into consideration. In their wide series of RFF experience, Sweeny et $\mathrm{al}^{9}$ did not use RFF for the scalp defects, which were more than $101 \mathrm{~cm}^{2}$. Such a limitation can be overcome by using another fasciocutaneous flap, ALT flap. Scalp reconstruction even with $420 \mathrm{~cm}^{2}$ ALT flap size has been recently reported. ${ }^{12}$ Its length of vascular pedicle can be safely 
extended with a meticulous dissection toward the origin of lateral circumflex femoral artery.

As previously described, ${ }^{1,13}$ LD flap was the most commonly used free flap option in our microsurgical scalp reconstruction series. The main difficulty that we experienced during the inset of the flap was the large muscle bulk and greater surface area of LD flap. We could perform skin-grafting of the rough surface during the primary surgery but we wanted to be sure of free flap survival. After the perioperative risk for microvascular complications was decreased, we performed the skin-grafting as a secondary procedure. We could also not incorporate a skin island to LD flap thereby harvesting a muscle-only flap. However, the skin island behaves like a monitor for flap survival and decreases the need for skin graft which may lead to postradiotherapy wound healing problems in this subset of high grade-malignancy patients. In addition, extended skin island design offers protection over the vascular pedicle itself. This design allows native skin island of flap to cover the microanastomoses and decreases the tension over the vascular pedicle. Otherwise it necessitates skin grafting, which leads to unsightly appearance and scar contracture.

Preoperative and postoperative radiation therapies were not found to be significantly associated with the development of complications although Chao et $\mathrm{al}^{14}$ found that a combination of the 2 was associated with increased late recipient site complications. Moreover, intense radiotherapy for the treatment of invasive skin cancer may cause fatal osteoradionecrosis of the skull, which is very rarely reported especially in the parietooccipital region. Once the diagnosis of osteoradionecrosis is established, aggressive radical surgery involving 3 important stages is mandatory to prevent further complications; eradication of infected and necrotic tissues, restoration of functioning viable tissues, and achieving an acceptable cosmetic result. ${ }^{15}$ Free flaps may not be readily used because the recipient vessels in the head and neck may be affected by the extensive radiation and previous surgeries as in the patient in Figure 2. Therefore, we had to explore the posterior neck to find suitable vessels. Transverse cervical vessels emerge as an excellent source of recipient in vessel-depleted head and neck. In addition, this maneuver removed us from traumatic zone of intense radiation.

Cranial bone reconstruction is important for the protection of the intracranial contents and for contouring the cranial vault. Cranial bone can survive even when it is not vascularized. Therefore, vascularized soft tissue is the key. Knowing the fact that reconstruction of the bony defect is not feasible without stable soft tissue coverage, ${ }^{16}$ our reconstruction algorithm avoids cranioplasty together with scalp and cranium reconstruction. Simultaneous cranioplasty during the immediate reconstruction has some controversies in the literature. Although the patients with cranial bone defect may suffer from headache, dizziness, loss of concentration, depression, anxiety, intolerance to noise, and vibration and neuromotor weakness, there are concerns for high rates of tumor recurrence, low survival rates, the need for postoperative radiotherapy, and the risk for surgical complications. ${ }^{17}$ Size and location of the bone defect, radiotherapy, and expected intracranial pressure are important determinants for considering bone reconstruction. Afifi et $\mathrm{al}^{18}$ performed a retrospective review of patients with simultaneous alloplastic cranioplasty and free flap reconstruction and reported a major complication rate of $38 \%$. Chao et al ${ }^{14}$ demonstrated that there was no significant difference between simultaneous or scalp-only reconstructions in terms of per operative complications although they observed a trend toward greater complications when cranioplasty was performed at the time of soft tissue reconstruction.
In conclusion, reconstruction of the extensive, composite scalp, and cranium defects with fasciocutaneous free flaps allows shorter hospitalization, less blood transfusion, and less flap atrophy than those of musculocutaneous flaps. That's why we recommend to use RFF in defects $<112 \mathrm{~cm}^{2}$ and to use ALT flap in defects $>112 \mathrm{~cm}^{2}$. Considering the extensive and destructive nature of the primary lesion, the necessity of multidisciplinary approach, which is an example of neuroplastic surgery, ${ }^{19}$ should be emphasized. The importance of transverse cervical vessels should also be underlined as recipient vessels in vessel-depleted head and neck.

\section{REFERENCES}

1. Sosin M, De la Cruz C, Bojovic B, et al. Microsurgical reconstruction of complex scalp defects: an appraisal of flap selection and the timing of complications. J Craniofac Surg 2015;26:1186-1191

2. Dalay C, Kesiktas E, Yavuz M, et al. Coverage of scalp defects following contact electrical burns to the head: a clinical series. Burns 2006;32:201-207

3. Mueller CK, Bader RD, Ewald C, et al. Scalp defect repair: a comparative analysis of different surgical techniques. Ann Plast Surg 2012;68:594-598

4. Beasley NJ, Gilbert RW, Gullane PJ, et al. Scalp and forehead reconstruction using free revascularized tissue transfer. Arch Facial Plast Surg 2004;6:16-20

5. Labow BI, Rosen H, Pap SA, et al. Microsurgical reconstruction: a more conservative method of managing large scalp defects? J Reconstr Microsurg 2009;25:465-474

6. Oh SJ, Lee J, Cha J, et al. Free-flap reconstruction of the scalp: donor selection and outcome. J Craniofac Surg 2011;22:974-977

7. Calikapan GT, Yildirim S, Akoz T. One-stage reconstruction of large scalp defects: anterolateral thigh flap. Microsurgery 2006;26:155-159

8. Kim SW, Hwang KT, Kim JD, et al. Reconstruction of postinfected scalp defects using latissimus dorsi perforator and myocutaneous free flaps. J Craniofac Surg 2012;23:1615-1619

9. Sweeny L, Eby B, Magnuson JS, et al. Reconstruction of scalp defects with the radial forearm free flap. Head Neck Oncol 2012;4:21

10. Simunovic F, Eisenhardt SU, Penna V, et al. Microsurgical reconstruction of oncologic scalp defects in the elderly. J Plast Reconstr Aesthet Surg 2016;69:912-919

11. Fischer JP, Sieber B, Nelson JA, et al. A 15-year experience of complex scalp reconstruction using free tissue transfer-analysis of risk factors for complications. J Reconstr Microsurg 2013;29:89-97

12. Kruse-Lösler B, Presser D, Meyer U, et al. Reconstruction of large defects on the scalp and forehead as an interdisciplinary challenge: experience in the management of 39 cases. Eur J Surg Oncol 2006;32:1006-1014

13. Lamaris GA, Knackstedt R, Couto RA, et al. The anterolateral thigh flap as the flap of choice for scalp reconstruction. J Craniofac Surg 2017;28:472-476

14. Chao AH, Yu P, Skoracki RJ, et al. Microsurgical reconstruction of composite scalp and calvarial defects in patients with cancer: a 10-year experience. Head Neck 2012;34:1759-1764

15. Nguyen MT, Billington A, Habal MB. Osteoradionecrosis of the skull after radiation therapy for invasive carcinoma. J Craniofac Surg 2011;22:1677-1681

16. Golpanian S, Kassira W, Habal MB, et al. Treatment options for exposed calvarium due to trauma and burns. J Craniofac Surg 2017;28:318-324

17. Rangan NM, Sahoo NK, Tomar K, et al. Efficacy of autogenous split thickness calvarial graft in the management of residual cranial defect. J Maxillofac Oral Surg 2015;14:754-760

18. Afifi AM, Djohan RS, Hammert W, et al. Lessons learned reconstructing complex scalp defects using free flaps and a cranioplasty in one stage. J Craniofac Surg 2010;21:1205-1209

19. Habal MB. Neuroplastic surgery: the new innovation in the educational process of craniofacial surgery. J Craniofac Surg 2018;29:1-3 